\title{
Metformin Treatment in Type 2 Diabetes in Pregnancy: An Active Controlled, Parallel-Group, Randomized, Open Label Study in Patients with Type 2 Diabetes in Pregnancy
}

\author{
Jahan Ara Ainuddin, ${ }^{1}$ Nasim Karim, ${ }^{2}$ Sidra Zaheer, ${ }^{3}$ \\ Syed Sanwer Ali, ${ }^{4}$ and Anjum Ara Hasan ${ }^{5}$ \\ ${ }^{1}$ Department of Obstetrics and Gynecology, Dow University of Health Sciences, Karachi 74400, Pakistan \\ ${ }^{2}$ Department of Pharmacology, Medical and Dental College, Bahria University, Karachi 75500, Pakistan \\ ${ }^{3}$ School of Public Health, Dow University of Health Sciences, Karachi 75300, Pakistan \\ ${ }^{4}$ Department of Community Health Sciences, United Medical and Dental College, Karachi 74900, Pakistan \\ ${ }^{5}$ Department of Obstetrics and Gynecology, Hamdard University Hospital, Karachi 74400, Pakistan
}

Correspondence should be addressed to Jahan Ara Ainuddin; jahanaraainuddin@yahoo.com

Received 14 December 2014; Revised 12 February 2015; Accepted 20 February 2015

Academic Editor: Vipin Gupta

Copyright (C) 2015 Jahan Ara Ainuddin et al. This is an open access article distributed under the Creative Commons Attribution License, which permits unrestricted use, distribution, and reproduction in any medium, provided the original work is properly cited.

\begin{abstract}
Aims. To assess the effect of metformin and to compare it with insulin treatment in patients with type 2 diabetes in pregnancy in terms of perinatal outcome, maternal complications, additional insulin requirement, and treatment acceptability. Methods. In this randomized, open label study, 206 patients with type 2 diabetes in pregnancy who met the eligibility criteria were selected from the antenatal clinics. Insulin was added to metformin treatment when required, to maintain the target glycemic control. The patients were followed up till delivery. Maternal, and perinatal outcomes and pharmacotherapeutic characteristics were recorded on a proforma. Results. Maternal characteristics were comparable in metformin and insulin treated group. 84.9\% patients in metformin group required add-on insulin therapy at mean gestational age of $26.58 \pm 3.85$ weeks. Less maternal weight gain $(P<0.001)$ and pregnancy induced hypertension $(P=0.029)$ were observed in metformin treated group. Small for date babies were more in metformin group $(P<0.01)$. Neonatal hypoglycemia was significantly less and so was NICU stay of $>24$ hours in metformin group $(P<0.01)$. Significant reduction in cost of treatment was found in metformin group. Conclusion. Metformin alone or with add-on insulin is an effective and cheap treatment option for patients with type 2 diabetes in pregnancy. This trial is registered with clinical trial registration number: Clinical trials.gov NCT01855763.
\end{abstract}

\section{Introduction}

Diabetes in pregnancy is becoming more common worldwide especially in South Asian countries like Pakistan. This is attributed to an increase in incidence of obesity and type 2 diabetes. Type 2 diabetes is a metabolic disorder characterized by hyperglycemia resulting from progressive deterioration in pancreatic insulin secretion, insulin resistance in tissues, and inadequate suppression of glucagon production [1]. Treatment of diabetes in pregnancy reduces serious perinatal morbidity, improves women's health related quality of life, and decreases maternal complications [2].
The increasing prevalence of type 2 diabetes in pregnancy brings with it specific challenges in terms of management. One such challenge that needs to be answered is, should metformin, an antihyperglycemic agent, be used in pregnancy with diabetes? Metformin is recommended as firstline treatment for patients with type 2 diabetes by American Diabetes Association [3]. However, metformin is not yet accepted as treatment for gestational diabetes and type 2 diabetes in pregnancy. Metformin offers a logical alternative to insulin in gestational diabetes and type 2 diabetes in pregnancy as it produces euglycemia by reducing insulin resistance, improving insulin sensitivity, reducing hepatic 
gluconeogenesis, and increasing peripheral glucose uptake and utilization. It also increases markers of endothelial activation which are intimately associated with insulin resistance [4-6]. Metformin, a category B drug, is not associated with fetal anomalies. Recent trial evidences support its safety and efficacy in pregnancy with gestational diabetes [7]. However as metformin crosses the placental barrier, fetal effects need to be considered [8]. Women with polycystic ovarian syndrome who continued on metformin throughout pregnancy showed decreased risk of miscarriages, preeclampsia, and gestational diabetes with better pregnancy outcomes and no increase in risk of congenital anomalies in newborns $[9,10]$. There is paucity of data in terms of clinical trials relating specifically to metformin treatment in women with type 2 diabetes in pregnancy. We have a population that brings specific challenges in terms of marked insulin resistance, poor compliance to insulin treatment, and lack of financial resources. Metformin instead of conventional insulin might be an alternative, effective, and cheap treatment option for women with type 2 diabetes in pregnancy in our population. The present study was aimed at evaluating the effects of metformin therapy in type 2 diabetes in pregnancy and comparing it with standard treatment insulin.

\section{Patients and Methods}

2.1. Study Design. This trial was a randomized open labeled clinical phase III study (NCT01855763) with parallel assignment to patients comparing metformin with insulin treatment in type 2 diabetes in pregnancy. The study was conducted at hospitals affiliated with Dow University of Health Sciences, Karachi, Pakistan, from January 2009 till January 2014. Institutional review board and ethics committee of the university approved the study and all participants gave informed written consent.

Patients with prepregnancy diagnosed type 2 diabetes and cases of newly diagnosed overt diabetes in pregnancy (IADPSG criteria: FBS: $\geq 7.0 \mathrm{mmol} / \mathrm{lit}(126 \mathrm{mg} / \mathrm{dL}), \mathrm{RBS}: \geq$ $11.1 \mathrm{mmol} / \mathrm{lit}(200 \mathrm{mg} / \mathrm{dL})$, and $\mathrm{HbAlC} \geq 6.5 \%)$ were selected from antenatal clinics after taking informed consent. They belonged to all five major ethnic groups living in urban and rural areas of four provinces in Pakistan but now settled in urban Karachi. Women included in the study were between 20 and 48 years of age, having a singleton pregnancy continued beyond first trimester. The exclusion criteria were women who have contraindications or intolerance to metformin intake like gastrointestinal side effects and altered liver functions with or without jaundice or had hypersensitivity with the drug and women who were diagnosed as gestational diabetes or had type 1 or type 2 diabetes and were already on insulin treatment, ultrasound showing a recognized fetal anomaly, ruptured membranes in second trimester, presence of any other medical disorder, and diabetes related complications.

Randomization was done as the eligible patients enter the study with odd number assigned to metformin treatment and even number for insulin treatment irrespective of body weight and previous obstetrical history. Blinding was not possible because of different routes of administration of drugs.

2.2. Study Treatment. Patients were advised for dietary modifications and nutritional instructions of three meals and three snacks daily with predesigned diets according to body weight. Metformin, (Glucophage) (Merck, Pakistan) was started at dose of $500 \mathrm{mg} /$ day orally and increased up to $2500 \mathrm{mg}$ in three divided doses as tolerated by the patient and till glycemic control was achieved. Target blood glucose levels for glycemic control were taken as fasting blood glucose (FBS) $\leq 100 \mathrm{mg} / \mathrm{dL}(5.5 \mathrm{mmol} / \mathrm{lit})$ and postprandial blood glucose levels (1.5 hours after meals) of $\leq 126 \mathrm{mg} / \mathrm{dL}$ ( $7 \mathrm{mmor} / \mathrm{lit})$ (Diabetic Association of Pakistan). If desired, target blood glucose levels were not maintained anytime during treatment even after maximum dose of metformin; insulin was added as supplementary treatment with metformin.

Insulin (Humulin R, Humulin N (Lilly)) was prescribed as a combination of short acting and intermediate acting human insulin as twice daily injections before meals in morning (before breakfast) and in evening (before dinner) to cover for the three meals and three snacks a day or as multiple injections of short acting insulin before meals and intermediate acting insulin at bedtime depending on individual patient requirement, in order to achieve the desired glycemic targets. Dose of insulin was calculated according to body weight and gestational age. A 24-hour total insulin dose was calculated using 0.6 units $/ \mathrm{kg}$ body weight in 1 st trimester, 0.7 units $/ \mathrm{kg}$ body weight in 2nd trimester, 0.8 units/kg body weight from 28 to 32 weeks of gestation, 0.9 units/kg body weight from 32 to 36 weeks of gestation, and 1 unit/kg body weight from 36 weeks onwards.

Patients were followed up in antenatal clinics. Iron, calcium, vitamin B12, and folic acid supplements were given to all patients. They were taught self, blood glucose monitoring using home glucose monitors and were advised to maintain a written or electronic record of blood glucose levels. Patients who could not monitor and record their blood glucose levels were tested using glucose monitors at each visit in antenatal clinic or they were admitted in day care ward for blood glucose monitoring when required. Fasting and three postprandial blood glucose levels 1.5 hours after breakfast, lunch, and dinner were recorded. Dose adjustments of drugs were made at each antenatal visit weekly or fortnightly till 36 weeks and then weekly till term or delivery. Routine obstetric care was provided at the antenatal clinics. HbAIC was done at study entry and at 36/37 weeks of pregnancy. Ultrasound was done at first visit (dating scan) then at 16-19 weeks (anomaly scan) and then monthly after 28 weeks (fetal well-being scan). Mode and time of delivery were decided at 37/38 weeks of pregnancy. Maternal and neonatal outcomes were recorded on a predesigned proforma.

\section{Study Outcome Measures}

The primary outcome measures were alive baby, neonatal hypoglycemia requiring intravenous dextrose therapy (blood glucose $<25 \mathrm{mg} / \mathrm{dL}$ or $<1.4 \mathrm{mmol} / \mathrm{lit}$ ), and NICU admissions $>24$ hours. 
The secondary outcome measures included birth weight, LGA/macrosomia (birth weight $>90$ centile for gestational age or birth weight $>4 \mathrm{~kg}$ ), SGA (birth weight $<10$ th centile for gestational age or birth weight $<2.5 \mathrm{~kg}$ ), neonatal morbidity like transient tachypnea of newborn, respiratory distress syndrome, prematurity, sepsis, jaundice, and birth trauma, maternal glycemic control reported as mean fasting (FBS) and postprandial blood glucose levels (RBS) and HbAIC levels at 36/37 weeks of pregnancy, weight gain in pregnancy, maternal hypertensive complications including pregnancy induced hypertension (gestational hypertension) and preeclampsia (defined as B.P $\geq 140 / 90$ on two occasions with significant proteinuria), and treatment compliance, dose, and cost of metformin and insulin treatment.

\section{Statistical Analysis}

4.1. Sample Size. It was estimated using the method of sample size estimation for comparing two means on www.openepi.com with mean and standard deviation of neonatal outcomes, hypoglycemia, and NICU admissions with an expected difference of 30\% reduction in NICU stay and $5-10 \%$ reduction in neonatal hypoglycemia between metformin and insulin treated groups from a study done in South Asian country [11] with 5\% margin of error and 95\% confidence interval. We worked on 206 samples. Using PASS version $11 \mathrm{Chi}$-square for proportion with 95\% CI, sample size of 206, and effect size of 0.233 with 2 degrees of freedom the power of study calculated is 0.86 from primary outcome measure, neonatal hypoglycemia, and for NICU admissions, the power of study calculated is 0.99 using effect size of 0.396 .

\section{Data Analysis}

Data was analyzed using IBM SPSS version 22; the frequencies and percentages were reported for all categorical variables. Mean with SD was reported for all continuous variables. The analysis was performed to compare the metformin alone group with metformin plus insulin group and insulin alone group using two-sample independent student $t$-test and Mann-Whitney $U$ test for continuous data. Chi square, Fisher Exact test, and Mann-Whitney $U$ test were used for categorical data. Two-tailed tests were used for all analysis and statistical significance was considered at $P \leq 0.05$.

\section{Results}

The overall design and subject flow through the study is illustrated in Figure 1. A total of 297 patients with type 2 diabetes in pregnancy were enrolled for the study. Of these 250 met the inclusion criteria and were randomized to treatment with metformin or insulin. 205 patients were already known cases of type 2 diabetes and were controlled on diet only or were on oral hypoglycemic agents. 45 patients were newly diagnosed cases and were classified as overt diabetes in pregnancy according to IADPSG criteria. 206 participants completed the study and their data was finally analyzed, with 106 patients in metformin group and 100 patients in insulin group (Figure 1). $84.9 \%$ patients in metformin group needed supplementary insulin treatment in varying doses to maintain glycemic control. The base line characteristics between the groups were not significantly different in metformin plus insulin and insulin alone groups; however patients in metformin alone group were younger and were of less parity (Table 1).

6.1. BMI and Weight Gain. BMI in early pregnancy was less in metformin alone group as compared to metformin plus insulin and insulin group $\left(28.25 \mathrm{Kg} / \mathrm{m}^{2}\right.$ versus $33.59 \mathrm{Kg} / \mathrm{m}^{2}$ versus $\left.32.96 \mathrm{Kg} / \mathrm{m}^{2}\right), P \leq 0.01$. Total weight gain in pregnancy was $10.38 \pm 1.2 \mathrm{~kg}$ in metformin alone group, $10.52 \pm$ $1.14 \mathrm{~kg}$ in metformin plus insulin group, and $11.80 \pm 0.86 \mathrm{~kg}$ in insulin alone group indicating significantly less weight gain in metformin treated group, $P \leq 0.01$ (Table 1).

6.2. Glycemic Profiles. Fasting, postprandial blood glucose levels and HbAIC levels were statistically comparable in three groups. Glycemic targets were achieved in all three groups within one week of starting treatment and maintained throughout pregnancy in the target range with no statistical difference in three groups (Table 1).

6.3. Maternal Outcomes. Pregnancy induced hypertension was found significantly less in metformin alone and metformin plus insulin group as compared to insulin alone group (6.2\% versus $23.3 \%$ versus $36 \%$ ), $P=0.020$. However the rates of preeclampsia in three groups were $25 \%$ versus $10 \%$ versus $17 \%, P=0.184$. All women who developed preeclampsia in metformin alone group were primigravidae. Gestational age at enrolment and at delivery was not significantly different in three groups. More women delivered vaginally in metformin plus insulin group. Vaginal delivery rates were $18.8 \%$ in metformin alone, $47.8 \%$ in metformin plus insulin group, and $18 \%$ in insulin group, $P \leq 0.01$. Cesarean section rate was $81.2 \%$ in metformin group, $52.2 \%$ in metformin plus insulin group, and $82 \%$ in insulin group showing significant reduction in cesarean section rate in metformin plus insulin group, $P \leq 0.01$ (Table 2).

6.4. Neonatal Outcomes. There were no perinatal deaths and all babies were born alive in three groups. Mean birth weights was statistically the same in all groups. Significantly more small for gestational age babies were found in metformin treated groups as compared to insulin alone group (31.2\% versus $14.4 \%$ versus $2 \%), P \leq 0.01$. Incidence of macrosomia was statistically the same in three groups $(12.5 \%$ versus $33.3 \%$ versus $27 \%$ ), $P=0.208$. Transient tachypnea of newborn was found in $12.5 \%$ versus $6.66 \%$ versus $18 \%$ in metformin alone, metformin plus insulin, and insulin alone group, $P=0.063$. Comparable rates of RDS were found in three groups with no statistical difference but lower rates in metformin plus insulin group (12.5\% versus $5.6 \%$ versus $11 \%), P=0.355$. $10 \%$ neonates in insulin alone group developed neonatal sepsis as compared to $3.3 \%$ in metformin plus insulin group, $P=0.094$. Two newborns in metformin alone group and 2 in insulin group had birth trauma with clavicle fracture in two neonates and cephalhematoma associated with vacuum delivery in other 2 neonates; all of them had birth weight of > $4 \mathrm{~kg}$. NICU stay was significantly less in metformin alone and 


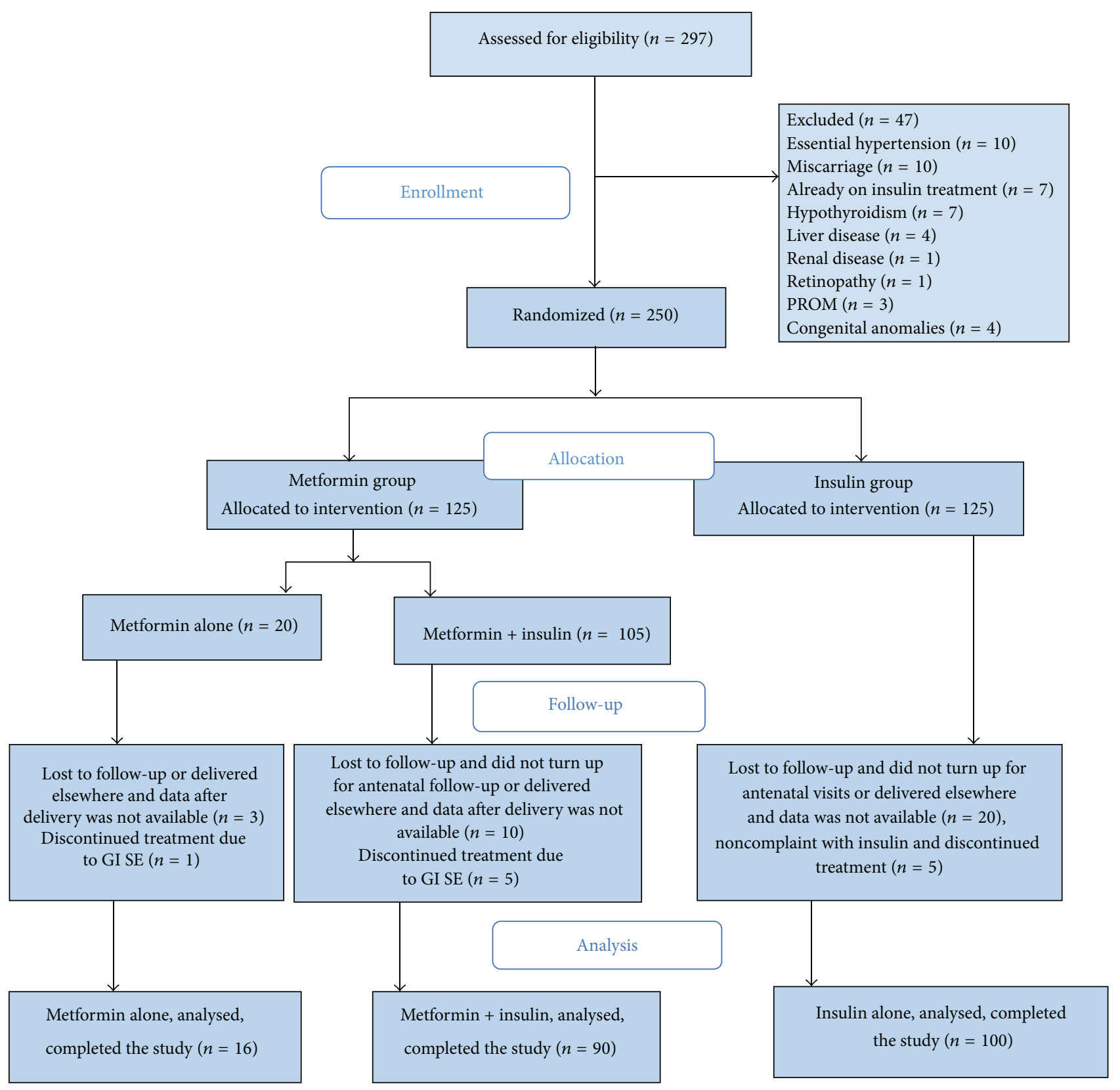

FIGURE 1: Flow diagram of study enrollment.

metformin plus insulin group as compared to insulin alone group, $P \leq 0.01$ (Table 3 ).

Significantly less neonatal jaundice was observed in metformin alone and metformin plus insulin group as compared to insulin group, $P=0.021$. Apgar scores at 5 minutes of birth were statistically comparable in 3 groups. Significantly less neonatal hypoglycemia was found in metformin plus insulin group, $P \leq 0.01$, and mean blood glucose levels at birth were also better in metformin plus insulin group (Table 3 ).

6.5. Pharmacotherapeutic Characteristics. Treatment compliance was good with oral metformin treatment than with insulin injections (see Supplementary Table in Supplementary Material available online at http://dx.doi.org/10.1155/ 2015/325851). Mean dose of metformin throughout pregnancy was $2.21 \pm 0.63$ in metformin alone group and $2.291 \pm 0.63$ grams per day in metformin plus insulin group, $P=0.665 .84 .9 \%$ patients required additional insulin in metformin group to maintain glycemic targets in mean dose of $23.64 \pm 4.61$ units per day. Mean dose of insulin required in insulin alone group was $77.62 \pm 12.69$ units per day which was significantly higher than that required in metformin group as add-on therapy, $P \leq 0.01$. Mean gestational age at which insulin was add-on in metformin group was $26.58 \pm 3.8$ weeks of pregnancy while insulin was started at mean gestational 
TABLE 1: Baseline demographic and maternal characteristics in treatment groups $(n=206)$.

\begin{tabular}{|c|c|c|c|c|c|}
\hline Parameters & Group & Mean \pm SD & $P$ value $^{\mathrm{a}}$ & $P$ value $^{\mathrm{b}}$ & $P$ value $^{\mathrm{c}}$ \\
\hline \multirow{3}{*}{ Age (years) } & Metformin alone & $31.75 \pm 2.82$ & $0.05^{*}$ & & \\
\hline & Metformin + insulin & $34.09 \pm 3.51$ & & 0.07 & \\
\hline & Insulin alone & $33.73 \pm 2.95$ & & & 0.956 \\
\hline \multirow{3}{*}{ Parity } & Metformin alone & $2.56 \pm 1.15$ & 0.550 & & \\
\hline & Metformin + insulin & $2.83 \pm 1.18$ & & $0.038^{*}$ & \\
\hline & Insulin alone & $3.18 \pm 1.15$ & & & $0.025^{*}$ \\
\hline \multirow{3}{*}{ BMI-early pregnancy $\left(\mathrm{kg} / \mathrm{m}^{2}\right)$} & Metformin alone & $28.25 \pm 1.98$ & $<0.01^{*}$ & & \\
\hline & Metformin + insulin & $33.59 \pm 3.97$ & & $<0.01^{*}$ & \\
\hline & Insulin alone & $32.96 \pm 4.04$ & & & 0.171 \\
\hline \multirow{3}{*}{ BMI-late pregnancy $\left(\mathrm{kg} / \mathrm{m}^{2}\right)$} & Metformin alone & $32.47 \pm 2.19$ & $<0.01^{*}$ & & \\
\hline & Metformin + insulin & $38.09 \pm 4.26$ & & $<0.01^{*}$ & \\
\hline & Insulin alone & $38.01 \pm 4.18$ & & & 0.714 \\
\hline \multirow{3}{*}{ Total weight gain in pregnancy $(\mathrm{Kg})$} & Metformin alone & $10.38 \pm 1.20$ & 0.868 & & \\
\hline & Metformin + insulin & $10.52 \pm 1.14$ & & $<0.01^{*}$ & \\
\hline & Insulin alone & $11.80 \pm 0.86$ & & & $<0.01^{*}$ \\
\hline \multirow{3}{*}{ FBS at starting treatment $(\mathrm{mg} / \mathrm{dl})$} & Metformin alone & $138.06 \pm 45.58$ & 0.304 & & \\
\hline & Metformin + insulin & $144.14 \pm 29.64$ & & 0.877 & \\
\hline & Insulin alone & $139.85 \pm 29.43$ & & & 0.155 \\
\hline \multirow{3}{*}{ RBS at starting treatment $(\mathrm{mg} / \mathrm{dl})$} & Metformin alone & $192.88 \pm 25.56$ & 0.326 & & \\
\hline & Metformin + insulin & $195.03 \pm 21.69$ & & $0.041^{*}$ & \\
\hline & Insulin alone & $201.21 \pm 16.78$ & & & 0.158 \\
\hline \multirow{3}{*}{ Mean FBS throughout pregnancy (mg/dl) } & Metformin alone & $97.87 \pm 3.83$ & 0.586 & & \\
\hline & Metformin + insulin & $97.50 \pm 3.35$ & & 0.672 & \\
\hline & Insulin alone & $97.55 \pm 3.29$ & & & 0.962 \\
\hline \multirow{3}{*}{ Mean RBS throughout pregnancy (mg/dl) } & Metformin alone & $136.79 \pm 5.34$ & 0.176 & & \\
\hline & Metformin + insulin & $134.79 \pm 5.69$ & & 0.237 & \\
\hline & Insulin alone & $135.31 \pm 4.64$ & & & 0.944 \\
\hline
\end{tabular}

$P$ value calculated by using independent $t$-test $\backslash$ Mann-Whitney $U$ test.

${ }^{a}$ Comparison between means of metformin alone and metformin + insulin groups.

${ }^{\mathrm{b}}$ Comparison between means of metformin alone and insulin alone.

${ }^{c}$ Comparison between means of metformin + insulin and insulin alone.

${ }^{*}$ Significant level $\leq 0.05$.

FBS: fasting blood glucose.

RBS: random blood glucose.

age of $9.55 \pm 5.2$ weeks in insulin group, $P \leq 0.01$. Cost of treatment was significantly less in metformin alone and metformin plus insulin group as compared to insulin alone group, $P \leq 0.01$ (Table 4 ).

6.6. Maternal and Neonatal Outcomes Adjusted for BMI. Out of 206 patients 50 had BMI $<30$ and 156 had BMI $>30$ indicating association of obesity with type 2 diabetes. Total weight gain in pregnancy was less in metformin alone group with $\mathrm{BMI}>30, P \leq 0.01$. Mean dose of add-on insulin was $27.63 \pm 6.03$ units in women with $\mathrm{BMI}<30$ and $22.84 \pm 3.78$ in women with $\mathrm{BMI}>30, P=0.519$, indicating no statistical difference in insulin dose, in groups stratified according to BMI. However mean gestational age at which insulin started was earlier in women with BMI > 30 as compared to women with $\mathrm{BMI}<30, P \leq 0.01$. Similarly pregnancy induced hypertension was less in women with $\mathrm{BMI}<30$ as compared to women with $\mathrm{BMI}>30, P=0.011$. There were no statistical differences in NICU stay and neonatal hypoglycemia in two groups stratified according to BMI, $P=0.249$ and $P=0.699$, respectively (Table 5).

\section{Discussion}

This randomized clinical study reports the results of metformin versus insulin treatment in women with type 2 diabetes in pregnancy. The study was conducted in a population setting with high prevalence of diabetes to meet the needs in a background of low economic resources where health facilities especially medicines are not always available free of cost. Patients with type 2 diabetes in pregnancy were selected with strict inclusion criteria; that is, they were not on insulin treatment and did not have complications associated with diabetes. Although duration of diabetes was not taken into account, the study patients, either had newly diagnosed overt diabetes detected in this pregnancy or were known 
TABLE 2: Maternal outcomes in treatment groups $(n=206)$.

\begin{tabular}{|c|c|c|c|c|}
\hline Parameters & $\begin{array}{c}\text { Metformin alone } \\
n=16 \\
n(\%)\end{array}$ & $\begin{array}{c}\text { Metformin }+ \text { insulin } \\
n=90 \\
n(\%)\end{array}$ & $\begin{array}{c}\text { Insulin alone } \\
\begin{array}{c}n=100 \\
n(\%)\end{array}\end{array}$ & $P$ value \\
\hline \multicolumn{5}{|c|}{ Maternal hypertensive complications in pregnancy } \\
\hline \multicolumn{5}{|c|}{ Pregnancy induced hypertension } \\
\hline Yes & $1(6.2)$ & $21(23.3)$ & $36(36.0)$ & \multirow{2}{*}{$0.020^{*}$} \\
\hline No & $15(93.8)$ & $69(76.7)$ & $64(64.0)$ & \\
\hline \multicolumn{5}{|l|}{ Preeclampsia } \\
\hline Yes & $4(25.0)$ & $9(10.0)$ & $17(17.0)$ & \multirow{2}{*}{0.184} \\
\hline No & $12(75.0)$ & $81(90.0)$ & $83(83.0)$ & \\
\hline \multicolumn{5}{|c|}{ Labor outcomes } \\
\hline \multicolumn{5}{|l|}{ Normal vaginal delivery } \\
\hline Yes & $3(18.8)$ & $43(47.8)$ & $18(18.0)$ & \multirow{2}{*}{$<0.01^{*}$} \\
\hline No & $13(81.2)$ & $47(52.2)$ & $82(82.0)$ & \\
\hline \multicolumn{5}{|l|}{ LSCS } \\
\hline Yes & $13(81.2)$ & $47(52.2)$ & $82(82.0)$ & \multirow{2}{*}{$<0.01^{*}$} \\
\hline No & $3(18.8)$ & $43(47.8)$ & $18(18.0)$ & \\
\hline \multicolumn{5}{|c|}{ Obstetric outcomes } \\
\hline & Mean \pm SD & $P$ value $^{\mathrm{a}}$ & $P$ value $^{\mathrm{b}}$ & $P$ value ${ }^{\mathrm{c}}$ \\
\hline \multicolumn{5}{|c|}{ Gestational age at enrolment (weeks) } \\
\hline Metformin alone & $10.75 \pm 5.98$ & 0.876 & & \\
\hline Metformin + insulin & $10.09 \pm 4.86$ & & 0.601 & \\
\hline Insulin alone & $9.57 \pm 5.20$ & & & 0.661 \\
\hline \multicolumn{5}{|c|}{ Gestational age at delivery (weeks) } \\
\hline Metformin alone & $36.19 \pm 1.68$ & 0.409 & & \\
\hline Metformin + insulin & $36.86 \pm 1.35$ & & 0.134 & \\
\hline Insulin alone & $37.06 \pm 1.22$ & & & 0.465 \\
\hline \multicolumn{5}{|c|}{$\begin{array}{l}P \text { value calculated by using Chi-square test } \backslash \text { Mann-Whitney } U \text { test. } \\
{ }^{a} \text { Comparison between means of metformin alone and metformin + insulin groups. }\end{array}$} \\
\hline
\end{tabular}

cases of type 2 diabetes, who were on diet control or on oral antihyperglycemic agents, with duration of diabetes not more than 5 years.

In mean age and BMI the difference was statistically insignificant in metformin and insulin treated groups but patients in metformin alone group were younger and most had $\mathrm{BMI}<30$. Older age and multiparity with $\mathrm{BMI}>30$ were also observed in previous studies as in our study [12-14]. Total weight gain in pregnancy was significantly less in metformin treated patients than in insulin treated patients. Similar results were reported by Rowan et al. [7, 15-17]. This effect of less weight gain was observed more in women with BMI > 30 in our study. Glycemic targets were achieved within one week of starting treatment and maintained throughout pregnancy. Similar findings were reported by Rowan et al., Hickman, Tertti, and others $[7,11,18-20]$ indicating metformin to be effective in achieving diabetes control.

We found significantly less pregnancy induced hypertension in metformin group as compared to insulin group, these findings being related to the effect of metformin on reducing endothelial activation and maternal inflammatory response to insulin resistance [21]. However more preeclampsia was found in elderly obese primigravidae in metformin alone group. No differences in rates of maternal hypertensive complications were found in other studies [7, 14, 16, 22, 23]. However, higher incidence of preeclampsia was reported by Hellmuth et al. in metformin treated patients [24]. Patients with $\mathrm{BMI}>30$ were found to have more pregnancy induced hypertension than patients with $\mathrm{BMI}<30$. Metformin treatment however is associated with significantly less occurrence of pregnancy induced hypertension in women with $\mathrm{BMI}>30$ in our study.

Patients were enrolled for study at around 10 weeks of gestation with a viable pregnancy. Mean gestational age at delivery was nearly the same around 37 weeks. More women delivered vaginally in metformin plus insulin group as compared to insulin alone group $(47.77 \%$ versus $18 \%)$ and similarly cesarean section rate was statistically lower in metformin treated group. No differences in cesarean section and vaginal delivery rates were reported in other studies [7, 13, 15, 24]. 
TABLE 3: Neonatal outcomes in treatment groups $(n=206)$.

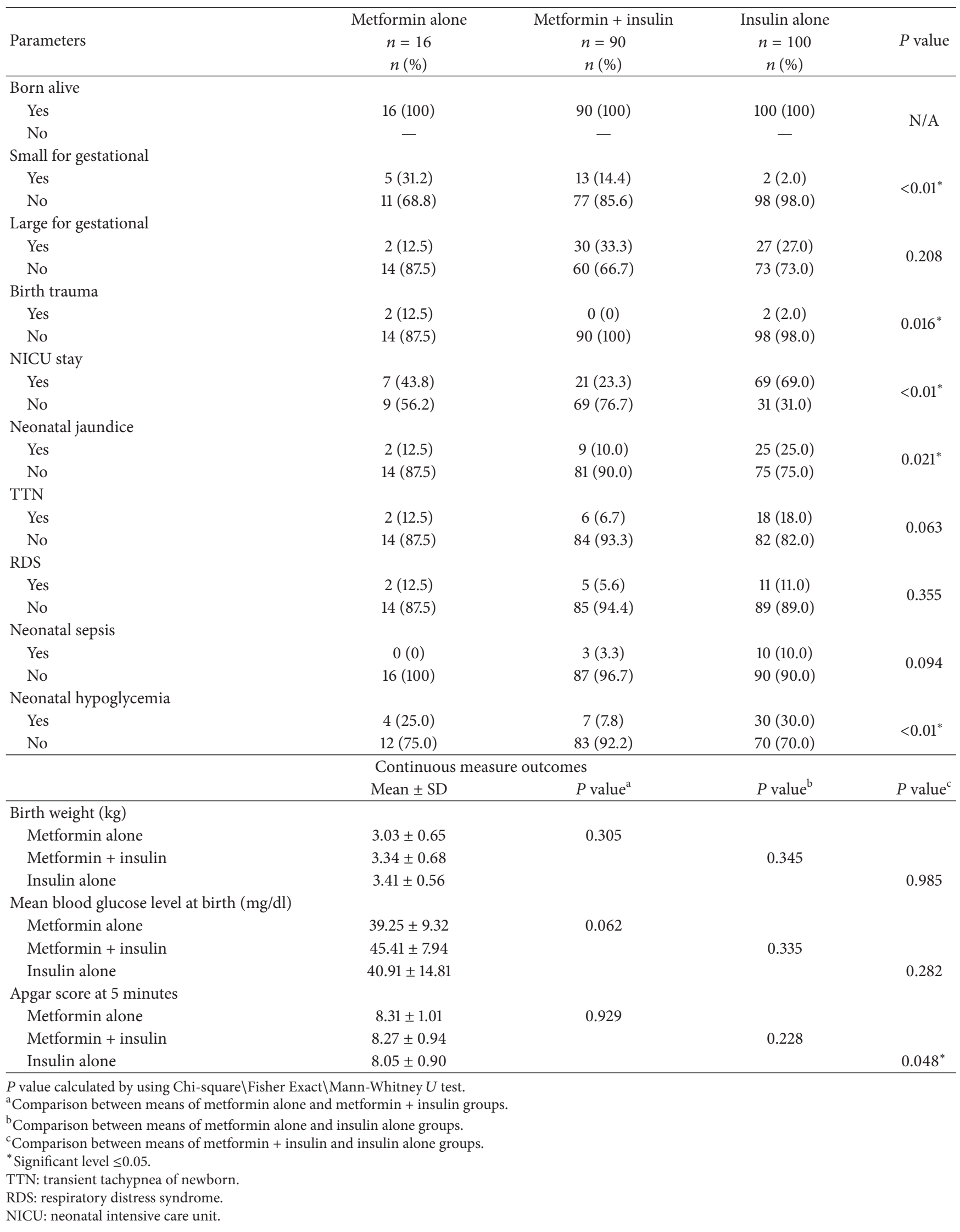


TABLE 4: Pharmacotherapeutic characteristics in treatment groups $(n=206)$.

\begin{tabular}{|c|c|c|c|c|c|}
\hline Parameters & Group & Mean \pm SD & $P$ value $^{\mathrm{a}}$ & $P$ value $^{\mathrm{b}}$ & $P$ value $^{\mathrm{c}}$ \\
\hline \multicolumn{6}{|c|}{ Dose of drug } \\
\hline \multirow{4}{*}{ Metformin: mean dose grams/day } & Metformin alone & $2.21 \pm 0.63$ & 0.665 & & \\
\hline & Metformin + insulin & $2.29 \pm 0.64$ & & - & \\
\hline & Insulin alone & - & & & - \\
\hline & Metformin alone & - & - & & \\
\hline \multirow[t]{3}{*}{ Insulin: mean dose units/day } & Metformin + insulin & $23.69 \pm 4.61$ & & - & \\
\hline & Insulin alone & $77.62 \pm 12.69$ & & & $<0.01^{*}$ \\
\hline & Metformin alone & - & - & & \\
\hline \multirow[t]{2}{*}{ Mean gestational age when insulin started (weeks) } & Metformin + insulin & $26.58 \pm 3.85$ & & - & \\
\hline & Insulin alone & $9.55 \pm 5.21$ & & & $<0.01^{*}$ \\
\hline \multicolumn{6}{|c|}{ Financial benefits } \\
\hline \multirow{4}{*}{ Total cost throughout pregnancy (PKR) } & Metformin alone & $448 \pm 131$ & $<0.01^{*}$ & & \\
\hline & Metformin + insulin & $5004 \pm 754$ & & $<0.01^{*}$ & \\
\hline & Insulin alone & $10976 \pm 6339$ & & & $<0.01^{*}$ \\
\hline & Metformin alone & $4.39 \pm 1.01$ & $<0.01^{*}$ & & \\
\hline \multirow[t]{2}{*}{ Total cost throughout pregnancy (USD) } & Metformin + insulin & $49.05 \pm 7.39$ & & $<0.01^{*}$ & \\
\hline & Insulin alone & $107.60 \pm 62.14$ & & & $<0.01^{*}$ \\
\hline
\end{tabular}

$P$ value calculated by using Mann-Whitney $U$ test.

${ }^{\text {a }}$ Comparison between means of metformin alone and metformin + insulin groups.

${ }^{\mathrm{b}}$ Comparison between means of metformin alone and insulin alone groups.

${ }^{\mathrm{c} C o m p a r i s o n}$ between means of metformin + insulin and insulin alone groups.

* Significant level $\leq 0.05$.

PKR: Pakistani rupee.

USD: US dollar.

However, $81.25 \%$ patients required cesarean section in metformin alone group mainly because of association with preeclampsia and intrauterine growth retardation.

No perinatal deaths occurred partly because congenital anomalies were excluded from the study and tight glycemic control was maintained, besides keeping a low threshold for cesarean section in cases of presumed fetal compromise. There was no difference in neonatal outcomes like birth weight, Apgar scores, large for gestational age infants, and respiratory distress syndrome in three groups. However significantly more small for gestational age infants were found in metformin group as compared to insulin group; association of preterm delivery before 37 weeks and birth weight $<10$ th centile for gestational age were contributing factors in metformin group. Rowan et al. reported more spontaneous preterm births in metformin group than in insulin treated group but same number of small for date infants in both groups in MIG trial [7]. Neonatal complications like transient tachypnea of newborn, neonatal sepsis, neonatal intensive care unit stay, and neonatal hypoglycemia were found significantly less in metformin treated group when compared with insulin group. Similar results were reported in other studies $[7,12,13,15,18,19,23]$. Women with BMI > 30 had more neonatal complications in insulin treated group as compared to metformin treated group indicating protective effect of metformin in reducing neonatal hypoglycemia and thus NICU admissions.

Patients achieved adequate glycemic control with mean dose of 2.5 grams of metformin, and few required dose limitation due to gastrointestinal side effects. These were those who required early add-on insulin therapy. Mean gestational age at which insulin started in metformin treated group was 26 weeks in our study. $84.9 \%$ patients in metformin group required add-on insulin therapy in our study. Patients with $\mathrm{BMI}>30$ required add-on insulin earlier than those with BMI < 30 in metformin treated group. BMI appeared to be a predictor for need of add-on insulin in metformin treated group, as patients who were controlled on metformin alone had mean BMI of less than 30. Insulin was add-on with metformin to maintain glycemic control when insulin resistance increases in pregnancy that is around 26-28 weeks of pregnancy. In metformin treated group, patients with early pregnancy BMI of $<30$ did not require add-on insulin or required add-on insulin at a later gestational age in our study. Ijas et al. reported similar results in their study [23]. Other studies in gestational diabetes show varying figures in terms of supplemental insulin requirement. Rowan et al. reported 46.3\%, Viollet et al. 18\%, and Moore et al. 34.7\% [7, 21, 25]. Coetzee and Jackson reported that $28.6 \%$ patients with type 2 diabetes in pregnancy required supplemental insulin with metformin [26]. Mohammed et al. in a retrospective analysis, however, reported that $95 \%$ patients with type 2 diabetes in pregnancy required additional insulin to maintain glycemic control [14]. These disparities in need for add-on insulin with metformin may be explained by differences in populations studied because diabetes and its control vary widely in different populations related to their genetic and phenotypic makeup. WHO ranks Pakistan as a high prevalent area for 


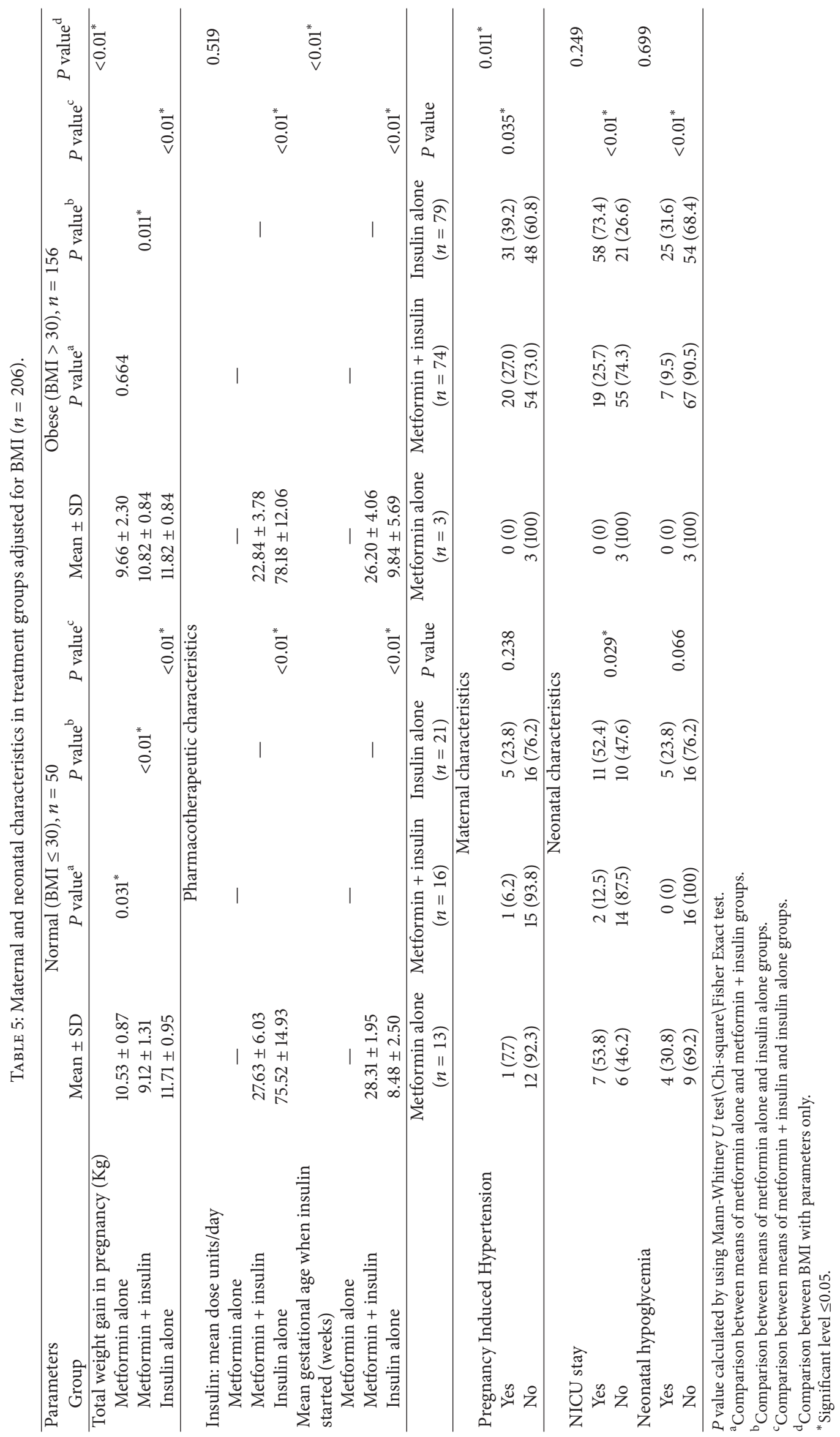


diabetes with patients exhibiting high insulin resistance and obesity [27]. This explains the reason of failure of metformin alone to achieve glycemic control in these patients. Mean dose of add-on insulin was significantly less in metformin group as compared to insulin group and so the financial benefit achieved was significant in metformin group. This financial benefit is also shown in gestational diabetes part of this study [28].

\section{Conclusion}

Metformin is an effective treatment option for women with type 2 diabetes in pregnancy with or without add-on insulin who require pharmacological treatment for glycemic control in our resource poor setting.

Metformin has advantages over insulin such as less maternal weight gain, no maternal hypoglycemia, being cheap, being oral therapy, and requiring no vigorous monitoring and frequent hospital admissions with good compliance and acceptability. Metformin treatment when compared with insulin treatment showed less maternal hypertensive complications and less risk of neonatal hypoglycemia with few neonatal intensive care admissions. Metformin treatment is suitable for nonobese type 2 diabetes patients in pregnancy without complications. Metformin treatment in type 2 diabetes in pregnancy required lower dose of add-on insulin, at a later gestational age for maintaining glycemic control when compared with insulin treatment.

Study limitations included duration of diabetes that was not taken into account in the present study; besides congenital anomalies were excluded from the study and longer infant follow-up was not carried out.

The findings of the trial are important in recommending metformin for treatment of type 2 diabetes in pregnancy in a selected population.

\section{Conflict of Interests}

The authors have no conflict of interests relevant to this paper.

\section{Authors' Contribution}

Jahan Ara Ainuddin designed and researched the study and wrote the paper. Nasim Karim wrote, reviewed, and edited the paper. Sidra Zaheer and Syed Sanwer Ali contributed to statistical analysis and edited the paper. Anjum Ara Hasan codesigned the study and edited the paper. Jahan Ara Ainuddin is the guarantor of this work, had full access of data in the study, and takes the responsibility for integrity and accuracy of data analysis.

\section{Acknowledgments}

The authors thank the study participants for their cooperation and regular follow-ups. The authors acknowledge the help of management of Mamji hospital and Dr. Khadim medical superintendent of Lyari General Hospital and their support in providing necessary equipment and medicines for the trial. Thanks are due to Dr. Ayesha Kamran, Dr. Lubna Rehman,
Dr. Moneeza, Dr. Tayyaba Anbareen, Dr. M. Yaqoob, and Dr. Kashif Abbas for their help in patient recruitment, data collection, and neonatal management.

\section{References}

[1] C. W. Spellman, "Pathophysiology of type 2 diabetes: targeting islet cell dysfunction," The Journal of the American Osteopathic Association, vol. 110, no. 3, pp. S2-S7, 2010.

[2] C. A. Crawther, J. E. Hiller, R. J. Moss et al., "Effect of treatment of gestational diabetes mellitus on pregnancy outcomes," The New England Journal of Medicine, vol. 352, no. 24, pp. 24772486, 2005.

[3] S. E. Inzucchi, R. M. Bergenstal, J. B. Buse et al., "Management of hyperglycaemia in type 2 diabetes: a patient-centered approach. Position statement of the American Diabetes Association (ADA) and the European Association for the study of diabetes (EASD)," Diabetologia, vol. 55, no. 6, pp. 1577-1597, 2012.

[4] G. Hawthorne, "Metformin use and diabetic pregnancy-has its time come?" Diabetic Medicine, vol. 23, no. 3, pp. 223-227, 2006.

[5] A. E. Caballero, A. Delgado, C. A. Aguilar-Salinas et al., "The differential effects of metformin on markers of endothelial activation and inflammation in subjects with impaired glucose tolerance: a placebo-controlled, randomized clinical trial," Journal of Clinical Endocrinology and Metabolism, vol. 89, no. 8, pp. 3943-3948, 2004.

[6] D. Kirpichikov, S. I. McFarlane, and J. R. Sowers, "Metformin: an update," Annals of Internal Medicine, vol. 63, pp. 1879-1894, 2002.

[7] J. A. Rowan, W. M. Hague, W. Gao, M. R. Battin, and M. P. Moore, "Metformin versus insulin for the treatment of gestational diabetes," The New England Journal of Medicine, vol. 358, no. 19, pp. 2003-2015, 2008.

[8] W. M. Hague, P. M. Davoren, D. McIntyre, R. Norris, X. Xiaonian, and B. G. Charles, "Metformin crosses the placenta," in Proceedings of the Medicine and Pregnancy, Novo Nordisk, Fremantle, Australia, October 2003.

[9] C. J. Glueck, N. Goldenberg, J. Pranicoff, M. Loftspring, L. Sieve, and P. Wang, "Height, weight, and motor-social development during the first 18 months of life in 126 infants born to 109 mothers with polycystic ovary syndrome who conceived on and continued metformin through pregnancy," Human Reproduction, vol. 19, no. 6, pp. 1323-1330, 2004.

[10] L. Harborne, R. Fleming, H. Lyall, J. Norman, and N. Sattar, "Descriptive review of the evidence for the use of metformin in polycystic ovary syndrome," The Lancet, vol. 361, no. 9372, pp. 1894-1901, 2003.

[11] S. Waheed, F. P. Malik, and S. B. Mazhar, "Efficacy of metformin versus insulin in the management of pregnancy with diabetes," Journal of the College of Physicians and Surgeons Pakistan, vol. 23, no. 12, pp. 866-869, 2013.

[12] R. C. E. Hughes and J. A. Rowan, "Pregnancy in women with type 2 diabetes: who takes metformin and what is the outcome?" Diabetic Medicine, vol. 23, no. 3, pp. 318-322, 2006.

[13] C. O. Ekpebegh, E. J. Coetzee, L. van der Merwe, and N. S. Levitt, "A 10-year retrospective analysis of pregnancy outcome in pregestational Type 2 diabetes: comparison of insulin and oral glucose-lowering agents," Diabetic Medicine, vol. 24, no. 3, pp. 253-258, 2007.

[14] M. A. Mohammed, A. M. Abdelmonem, M. A. Abdullah, and A. A. El Sayed, "Oral hypoglycemic as attractive alternative 
to insulin for the management of diabetes mellitus during pregnancy," Gynecology \& Obstetrics, vol. 4, no. 1, article 193, 2014.

[15] J. Balani, S. L. Hyer, D. A. Rodin, and H. Shehata, "Pregnancy outcomes in women with gestational diabetes treated with metformin or insulin: a case-control study," Diabetic Medicine, vol. 26, no. 8, pp. 798-802, 2009.

[16] C. P. Spaulonci, L. S. Bernardes, T. C. Trindade, M. Zugaib, and R. P. V. Francisco, "Randomized trial of metformin vs insulin in the management of gestational diabetes," American Journal of Obstetrics and Gynecology, vol. 209, no. 1, pp. 34.e1-34.e7, 2013.

[17] L. Rai, D. Meenakshi, and A. Kamath, "Metformin-a convenient alternative to insulin for Indian women with diabetes in pregnancy," Indian Journal of Medical Sciences, vol. 63, no. 11, pp. 491-497, 2009.

[18] M. A. Hickman, R. McBride, K. A. Boggess, and R. Strauss, "Metformin compared with insulin in the treatment of pregnant women with overt diabetes: a randomized controlled trial," The American Journal of Perinatology, vol. 30, no. 6, pp. 483-489, 2013.

[19] K. Tertti, U. Ekblad, T. Vahlberg, and T. Rönnemaa, “Comparison of metformin and insulin in the treatment of gestational diabetes: a retrospective, case-control study," Review of Diabetic Studies, vol. 5, no. 2, pp. 95-101, 2008.

[20] M. I. Ibrahim, A. Hamdy, A. Shafik, S. Taha, M. Anwar, and M. Faris, "The role of adding metformin in insulin-resistant diabetic pregnant women: a randomized controlled trial," Archives of Gynecology and Obstetrics, vol. 289, no. 5, pp. 959-965, 2014.

[21] B. Viollet, B. Guigas, N. S. Garcia, J. Leclerc, M. Foretz, and F. Andreelli, "Cellular and molecular mechanisms of metformin: an overview," Clinical Science, vol. 122, no. 6, pp. 253-270, 2012.

[22] K. Swaminathan, H. C. S. Howlett, and I. W. Campbell, "Metformin in diabetic pregnancy," Journal of the Royal College of Physicians of Edinburgh, vol. 39, no. 1, pp. 10-14, 2009.

[23] H. Ijas, M. Vaarasmaki, and L. Morinpaunen, "Metfromin should be considered in the treatment of gestational diabetes: a prospective randomized study," BJOG, vol. 10, 2010.

[24] E. Hellmuth, P. Damm, and L. Mølsted-Pedersen, "Oral hypoglycaemic agents in 118 diabetic pregnancies," Diabetic Medicine, vol. 17, no. 7, pp. 507-511, 2000.

[25] L. E. Moore, G. I. Brieny, and D. Clokey, "Metformin and insulin in the management of gestational diabetes mellitus," The Journal of Reproductive Medicine, vol. 52, pp. 1011-1015, 2007.

[26] E. J. Coetzee and W. P. U. Jackson, "Pregnancy in established non-insulin-dependent diabetics. A five-and-a-half year study at Groote Schuur Hospital," South African Medical Journal, vol. 58, no. 20, pp. 795-802, 1980.

[27] Clinical Guidelines Task Force and International Diabetes Federation, Global Guidelines for Type 2 Diabetes, 2009, http://www.idf.org/.

[28] J. Ainuddin, N. Karim, A. A. Hasan, and S. A. Naqvi, "Metformin versus insulin treatment in gestational diabetes in pregnancy in a developing country. A randomized control trial," Diabetes Research and Clinical Practice, vol. 107, no. 2, pp. 290299, 2015. 


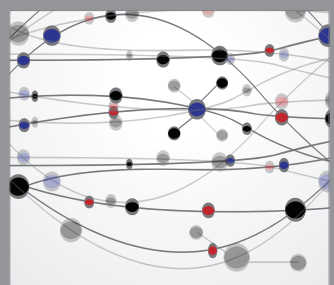

The Scientific World Journal
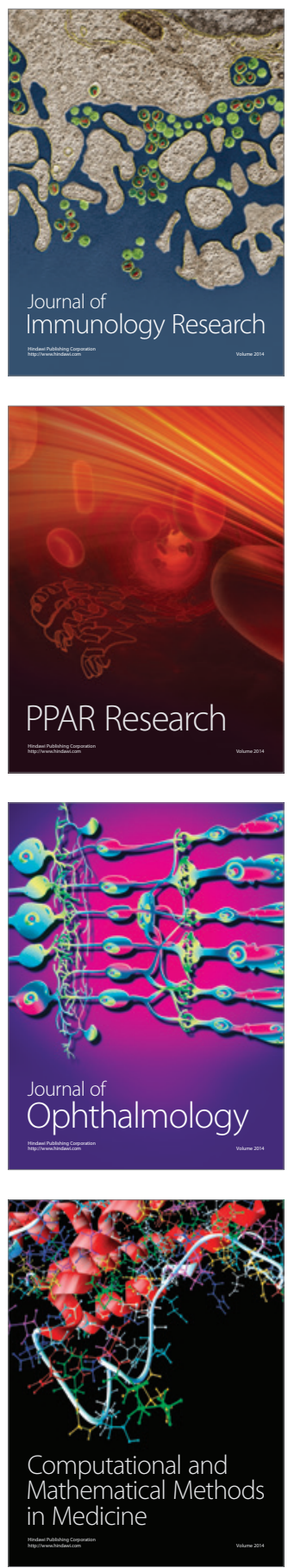

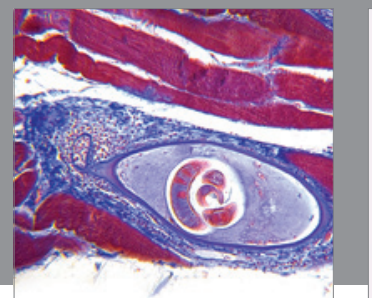

Gastroenterology

Research and Practice
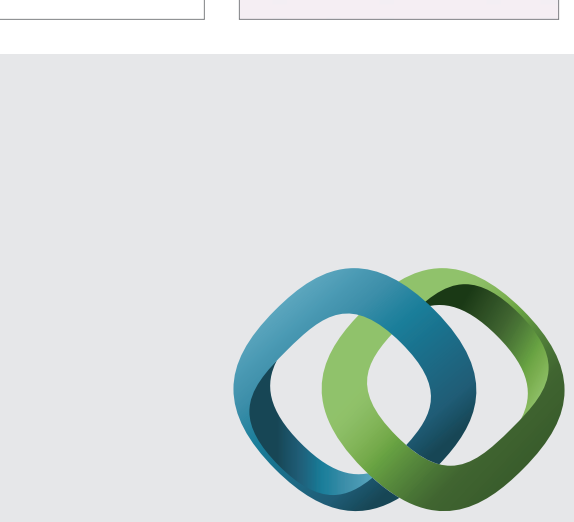

\section{Hindawi}

Submit your manuscripts at

http://www.hindawi.com
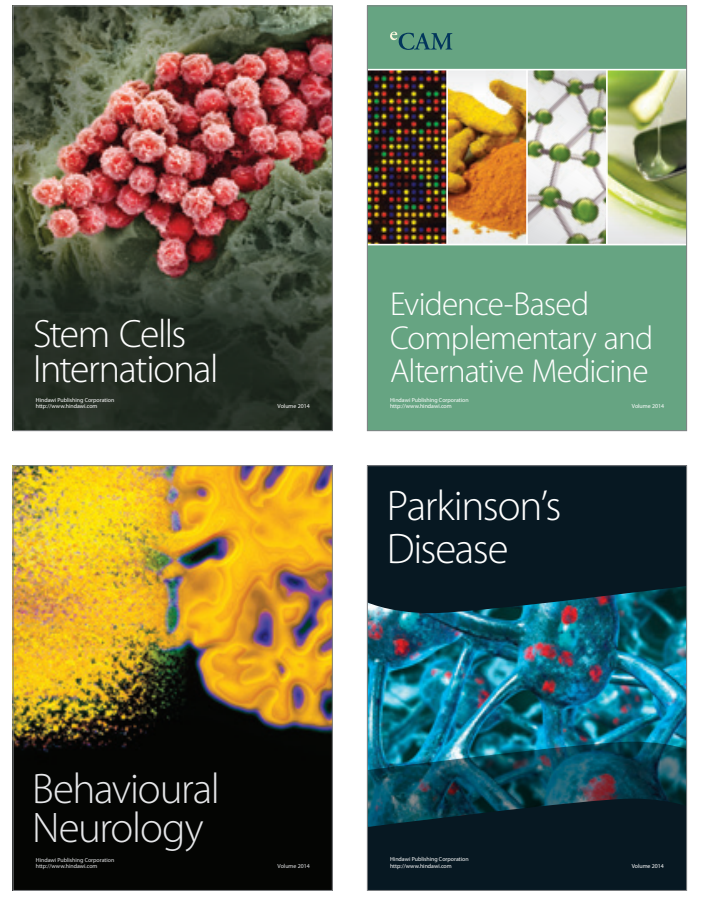
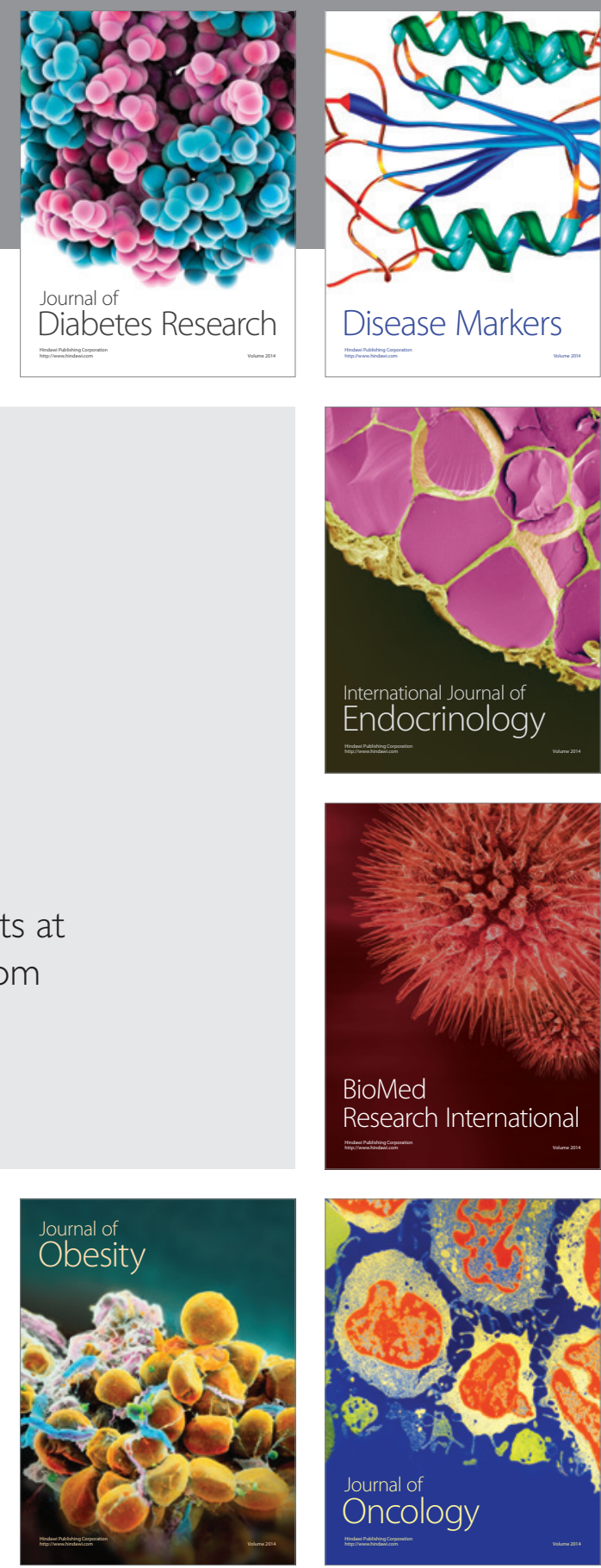

Disease Markers
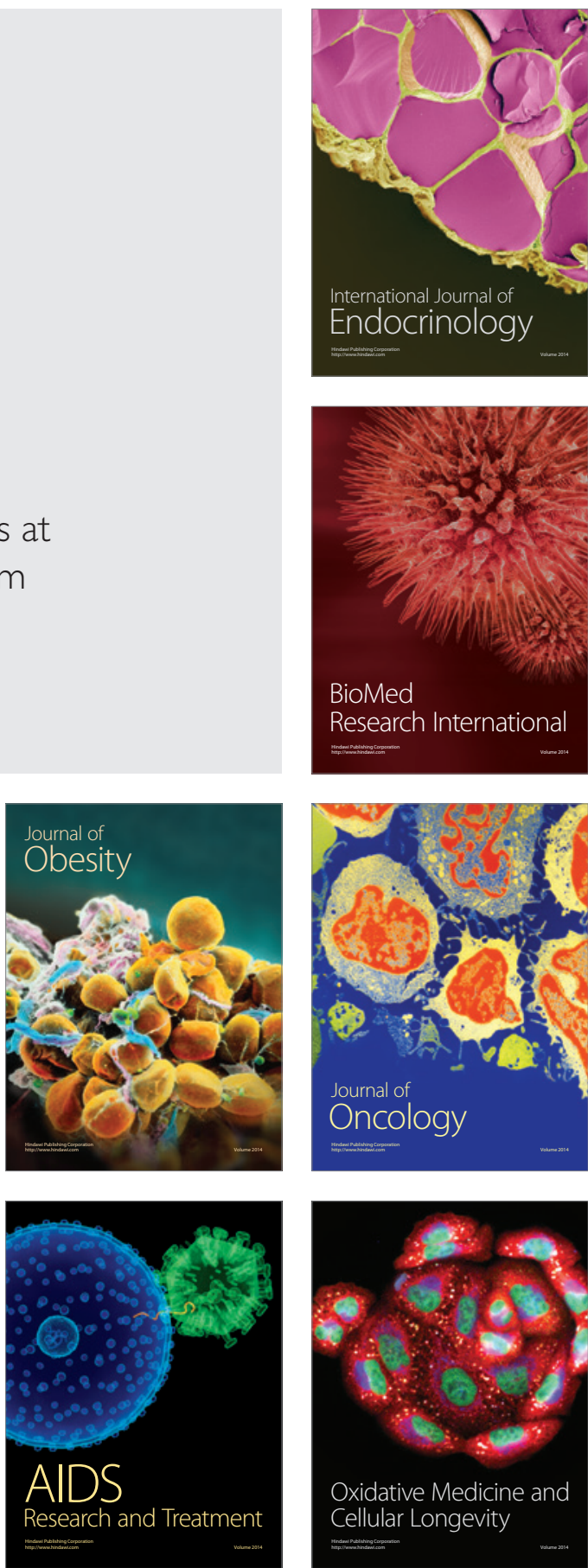